\title{
ON PHILOSOPHICAL ANTHROPOLOGY AND PSYCHOLOGY
}

M.Ch. Michailov 1, E. Neu 1, U. Welscher 1, P. Birkenbihl 1, Ch. Luetge 2, G. Weber 3

1 Inst. Umweltmedizin c/o ICSD e.V., Munich, Germany.

2 Techn. Univ. Muenchen, Inst. Ethics, Dir., Munich, Germany.

3 Univ. Lxbg. \& Vienna, Fac. Psychol., Dean, Vienna, Austria.

INTRODUCTION: Considering the fundamental question of philosophy: "What is the human?" Immanuel KANT created scientific philosophical anthropology. This is essential for a future integrative psychology. New interpretation of psychology related to occidental-oriental philosophy and medicine is necessary, beginning with ARISTOTELES-PLATONTHOMAS VON AQUIN-DESCARTESLEIBNIZ, etc. as well as AVICENNAHIPPOCRATES-GALENUS-HUA T'UAPARACELSUS.

\section{CONCEPTION for discussion:}

A. EPISTEMOLOGY. Creation of an integral anthropology (individual-natural-social) including holistic \& multidimensional information about human being (spiritualmental and other upto somatic "spheres") \& interaction with nature (geo-biospheres) \& society (family, school, etc.) related to psychology in context of psychosomatic (Th.von UEXKÜLL) \& somatopsychic theories (Y.IKEMI) is conditio sine qua non for future education ("Bildung \& Erziehung").

B. MORAL PHILOSOPHY. Ethical-frames destination acc. to KANT's human obligations to himself (psychologists and personnel), other humans (patients), sub-humans (e.g. reduction of animal experiments), suprahuman beings (patients from different cultures: Brahmanism, Buddhism-Shintoism, Christianism-Mosaism, Confucianism-Taoism, Mohammedanism, etc.) related to modern ethical theories (deontology, utilitarism, etc.) are fundamental for future psychology.
C. AESTHETICS. Psychotherapy \& psychopharmacological-surgical-treatment must be in concordance with ethics in psychology and medicine (primum non nocere), e.g. for oncological (radiochemotherapy) or dental patients (infection of teeth, etc.), in context of psychooncology \& psychoodontology. Selfcontrol of humans incl. patients by occidental (cognitive therapy, etc.) and oriental practices (Buddhist, Yoga, etc.) could counteract psychopathological \& pathophysiological reactions supporting total health (spiritual, mental, social, etc.) of patients.

CONCLUSION: Foundation of an European and an International Academy for Psychology, similar to European Acad. Neurology (EAN-2015-Berlin), by EFPA/IUPsyS/American/European incl. Russian societies, e.g. in MoscowKaliningrad or West-Europe could support conception (A-C) in context of UNOAgenda21 for better health-ecology-economy on global level.

\section{A. EPISTEMOLOGY Fig. 1}

[Ref.B: Th.von Uexküll]

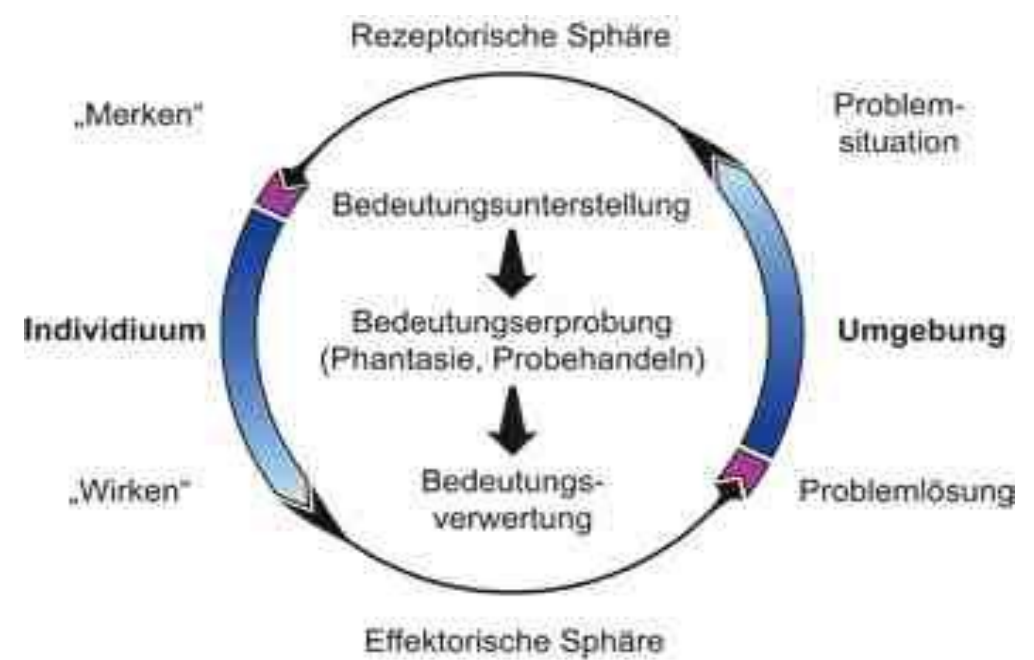

B. MORAL PHILOSOPHY

Fig. 2

Model about

thics in context of an integral anthropology

(Related to Human Obligations acc. to Kant and Dimensions acc. to Jannsens)

$\begin{array}{ll}\text { Pedagogical } & \begin{array}{l}\text { General Anthropology } \\ \text { Philosophical } \\ \text { Educative }\end{array} \\ \text { Normative }\end{array}$

Medical

Educative

Normative

Prophylactic, Curative

Himself

Special Anthropology

Anthropology:

Individual

Human Dimensions:

Social

$A+\mathrm{G}+\mathrm{H}+\mathrm{X}$

Natural

$B+C+X 3$

Supra-Human (God, etc.) Supra-Natural $\quad F+X 4(X n=V a r i a)$

Louis Jannsens (ref. B: Book "European Bioethics" 2007) described 8 dimensions of human person, i.e. relations person (see Table)
A Subject
B. Body
C. Material World
D. Other Persons
E. Social World F. God
G. Historical Being
$\mathrm{H}$. Unique/Equal to others

Immanuel Kant (Metaphysik der Sitten): Human obligations to Human, i.e. to himself $(1 a)$ and other human beings $(1 b)$ as well as non-human beings, i.e. subhuman (2a: plants, animals, etc.) and supra-human (2b: supernal world, God). This is related to Jannsens considerations.

Kant summarized obligations in context of moral philosophy and law.

\section{$\begin{array}{lll}\text { C. AESTHETICS } & \text { Fig. } 3 \quad \text { [see Neu, Michailov et al. this congress] }\end{array}$}

References: Michailov, Neu et al. Philosophy: FISP-2018-Peking (World-Congr.Philos.) (inpress); -2013-Athens, Abstr.-Book (AB):464-5\&503-4\&766. -2008/10-Seoul Proc.DVD/ISBN-13, 195-202/338-339/363-365; -2003-Istanbul AB:279-280. EACME-2017-Barcelona, Congr.Book, ed. Inst.Bioetica/Univ.Ramon-Llull, p.73-74\&125-126. ESPMH-2010-Zagreb, AB:46-47. IAB-2018India (Bioethics, in-press); -2016-Edinburgh, AB:93-95; -2008-Istria/Croatia, AB:290\&307. Psychology/Psychiatry: IUPsyS-2012-Cape-Town, IntJPsychol 47:407; further see Neu et al./EFPA/ECP-2019. WPA-2018-Mexico (in-press); -2011-Buenos-Aires, AB:PO1.200. EPA-2018Nice, Eur. Psychiatry 48/S1:S623\&567\&662. Physiology/Psychosomatics: IUPS-2017-Rio-deJaneiro, AB:No. 997,999,1001,1003; -2009-Kyoto. J.Physiol.Sci., 59/S1:168\&214\&447-8. -1997St.Petersburg AB:P036.02/.03/.04/P058.38. ICPM-2017-Beijing, AB:ID: 648493, 648895, 648749, 648878; -2011-Seoul, AB:167,186; -2005-Kobe, J.Psychosom.Res. 58:85-86.

DEDICATION [see Neu, Michailov et al. this congress] 